\title{
Kalziumphosphatverbindungen zum Knochenersatz
}

\author{
$\square$ Johannes M. Rueger, Andreas Rücker, Arnd Schilling, Daniel Briem
}

\section{Zusammenfassung}

Für die Behandlung von Defekten im Knochen, unabhängig von der Genese, und bei dem Versuch, Knochenheilung zu „Biologisieren“, stehen neben dem autogenen Knochen unterschiedliche Substanzen zur Auswahl. Eine Gruppe von Substanzen sind die Kalziumphosphate, die in unterschiedlichen molaren Verhältnissen zwischen Kalzium und Phosphat hergestellt werden können. Dieses stöchiometrische $\mathrm{Ca} / \mathrm{P}-$ Verhältnis beeinflusst massiv das biologische Verhalten der Materialien nach dem Einbringen in den Organismus und ist letztendlich für die zelluläre Resorption bzw. die physikochemische Degradation - und somit auch für den Ein- bzw. Abbau am Implantationsort - verantwortlich. Die bis heute benutzten Substanzen aus der Gruppe der Kalziumphosphate, die Materialien mit denen experimentell und klinisch die größten Erfahrungen gewonnen werden konnten, sind Hydroxylapatit- (HA) und Trikalziumphosphatkeramiken (TCP), wobei letztgenannte sowohl in ihrer Hochtemperaturphase $=$ alpha-TCP als auch in ihrer Niedrigtemperaturphase = beta-TCP eingesetzt werden. In Abhängigkeit von der Indikation als auch dem Implantationsort lassen sich heute eindeutige, gute Indikationen für den Einsatz von HA und TCP empfehlen, wobei auf einige Grundregeln zu achten ist, um das Auftreten von Komplikationen zu vermeiden und den angestrebten klinischen Erfolg für den Patienten zu erreichen.

\section{Calcium Phosphate Compounds as Bone Substitutes}

In the treatment of bony defects, irrespective of their cause and in attempts to "biologise" and or "activate" bone healing numerous different substances have been recommended as substitute materials for autogenous bone. Calcium phosphate compounds are a group of materials defined according to their molar $\mathrm{Ca} / \mathrm{P}$ ratio. The latter massively influences the biological reaction after implantation of such materials since it is responsible for the degree of cellular resorption and/or physicochemical degradation, thus determining the amount of non-integration or integration at the implantation site. Well known materials among the calcium phosphate compounds, materials that have been extensively experimentally investigated and clinically applied, are hydroxyapatite (HA) ceramics and tricalcium phosphate (TCP) ceramics, the latter sintered either at about $800{ }^{\circ} \mathrm{C}$ (beta-TCP) or above $1250{ }^{\circ} \mathrm{C}$ (alpha-TCP). Depending on the indication as well as the implantation site, we are today able to give sound recommendations either for the implantation of HA or TCP and, if some general rules are observed, material-related complications can be avoided with excellent clinical results for our patients.

\section{Einleitung}

Die Ursachen für die Entstehung von knöchernen Defekten sind vielfältig. Trauma führt zu diaphysären Trümmerzonen (z.B. Humerusschaft, Unterarm, Femur-/Tibiaschaft), Defekten mit knöchernen Substanzverlusten (z.B. offene Frakturen Tibiaschaft, Mehretagenfrakturen dort) und Impressionszonen im epimetaphysären Bereich (z.B. Humeruskopf, distaler Radius, Tibiakopf, Pilon tibiale). In Abhängigkeit vom Frakturtyp, abhängig von der angewandten OPTechnik bzw. beim Auftreten vom Komplikationen können aus zunächst begrenzten Defekten ausgedehnte werden, die in der Folge ein Auffüllen der Defektzonen bei der Therapie erforderlich machen.

Weitere Ursachen für die Entstehung von ossären Defekten sind Knocheninfektionen, Resektionen bei der Tumor-/Metastasenchirurgie bzw. Knochenmasseverluste nach endoprothetischer Versorgung zum Zeitpunkt der Revisionsendoprothetik.

Unsere Möglichkeiten, langstreckige Defekte zu überbrücken, sind durch die Ilisarov-Technik (Segmenttransport), Technik der primären Verkürzung und sekundären Verlängerung durch die Distraktionsosteogenese, durch die Transplantation mikrovaskulär angeschlossener, freier Fibulae bzw. Beckenkammtransplantate schon vor Jahren deutliche verbessert bzw. revolutioniert worden. Mit der Einführung geschlossener Repositionstechniken, minimalinvasiver Verfahren (Technik der eingeschobenen Platte), winkelstabiler Plattensysteme und neuer Nageltechnologien, die es erlauben, auch sehr metaphysennah gelegene Frakturen sicher über eine Nagelosteosynthese „gedeckt“ zu stabilisieren, ist erfreulicherweise die Inzidenz der durch offene Osteosyntheseverfahren verursachten Komplikationen massiv rückläufig. Offene Verfahren der Reposi- 
tion und Stabilisierung gingen - und gehen - immer mit dem Risiko der Devaskularisierung von Fragmenten und - bei intraoperativen Komplikationen - auch mit der Entstehung von Infekten einher, als deren Folgen zunächst Frakturheilungsstörungen, im schlimmsten Fall jedoch die Entstehung von knöchernen Defekten zu beobachten sind.

\section{Knochendefektbehandlung, Biologi- sierung der Fakturheilung: Standard}

Für die Knochendefektbehandlung wird weiterhin autogener Knochen (in der Form der autogenen Spongiosaplastik, als kortikospongiöse Chips, bi-/trikortikale Späne) als goldener Standard - und dies sicherlich immer noch zu Recht verstanden. Langstreckige Substanzverluste im diaphysären Bereich können heute mit der bereits oben erwähnten Distraktionsosteogenese sicher überbrückt werden, so dass dort das Verfahren einer alleinigen, autologen Spongiosatransplantation zumindest selten, wenn nicht obsolet geworden ist.

Durch den Einsatz winkelstabiler Implantate, Formplatten in Abhängigkeit von der Frakturlokalisation/-region und - wiederum - spezieller Nägel können heute auch epimetaphysäre Frakturen am Humeruskopf, distalen Radius, distalen Femur, Tibiakopf, Pilon tibiale und am Kalkaneus unter bestimmten Indikationen bzw. bei bestimmten Frakturtypen ohne das Auffüllen der metaphysären Defekte adäquat stabilisiert und zur sicheren Ausheilung gebracht werden. Trotz dieses Erfolges muss jedoch realistisch gesehen werden, dass autogene Knochen-, speziell Spongiosatransplantationen regelmäßig nötig sind und eingesetzt werden bei:

- Wirbelsäulenfusionsoperationen

- nicht Osteoporose assoziierten Humeruskopffrakturen,

- distalen, intraartikulären Humerusfrakturen,

- distalen Radiusfrakturen mit ausgedehnten Gelenkimpressionszonen,

- distalen supra- und intrakondylären Femurfrakturen,

- Tibiakopffrakturen(Spalt-/Impressionbrüche) und

- Pilon-tibiale-Frakturen.

Dieses relativ begrenzte Spektrum für den Einsatz autogenen Knochens erweitert sich rasch, wenn die therapeutischen Möglich- bzw. Notwendigkeiten bei der Behandlung von Komplikationen der Knochenheilung (verzögerte Heilung,

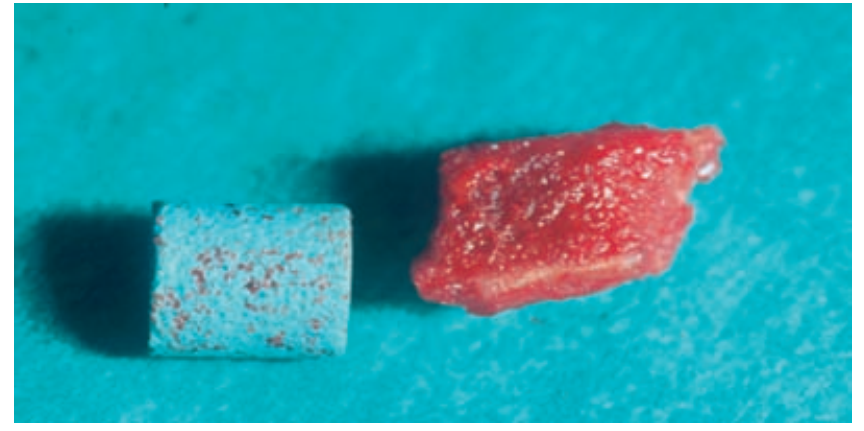

Abb. 1 Fotografie. Makroporöser HydroxylapatitkeramikZylinder versus segmentalem Resektat aus Hundeulna.

atrophe Pseudarthrosenbildung, Infektsanierung) einbezogen werden.

Hinzu kommen zahlreiche Indikationen, bei denen in Abhängigkeit von der Frakturform und -lokalisation, Weichteilsituation und Alter des Patienten eine „Biologisierung“ der Fraktur, insbesondere nach ORIF wünschenswert erscheint.

\section{Als Beispiele:}

- Klavikulaschaftfraktur in Schaftmitte, disloziert, mindestens zwei kleine Drehkeile;

- distale Humerusfraktur, Defekte zwischen ulnarem/radialem Pfeiler;

- Ulnafraktur, mehrere kleine Drehkeile;

- usw.

Wenn autogene Spongiosa auch der goldene Standard ist, so erfüllt sie jedoch aus zahlreichen Gründen nicht alle der an sie gestellten Erwartungen. So gibt es zahlreiche Probleme bei der Gewinnung (zweiter OP-Situs, Kostenaufwand, begrenzte Menge insbesondere bei Kindern, Qualität des zu gewinnenden Knochens und postoperative Schmerzen, mögliche Wundheilungsstörungen, sekundäre Beckenfrakturen bei ausgedehnten Spongiosa-Entnahmen).

Bei kleinen Defekten muss weiterhin die Indikationsstelle zur Knochenentnahme deutlich gegen die möglichen Risiken abgewogen werden. Auch autogene Spongiosatransplantationen haben eine Versagerquote und sind kein Allheilmittel bei Infekten bzw. indikatorischen oder OP-technischen Fehlern. Völlig überfordert sind die Möglichkeiten der Knochentransplantation bei Magnum-Defekten, verursacht zum Beispiel durch Kopf-Nekrosen (Humerus, proximaler Femur), bikondylären Zertrümmerungen (distaler Femur, Tibiakopf), ausgedehnten Becken-/Azetabulum-Pseudarthrosen, in der Tumorchirurgie und Revisions-Endoprothetik.

\section{Knochenersatzstoffe: Anforderungen, Klassifikation}

Die zurzeit verfügbaren und regelmäßig eingesetzten Alternativen für das autogene Knochentransplantat bei der Behandlung von Knochendefekten, bei der Rekonstruktion zerstörter Gelenkflächen bzw. bei Fusionsoperationen an der Wirbelsäule sind die Distraktionsosteogenese am diaphysären Röhrenknochen, der endoprothetische Gelenkersatz und metallische/Kunststoffimplantate („Cages“) in der Fusionschirurgie mit reproduzierbar sehr guten bzw. guten Ergebnissen.

Für alle anderen oben aufgeführten Indikationsbereiche stehen Knochenersatzstoffe („Bone Substitutes“) = Knochenersatzmaterialien („Bone Replacement Materials“) zur Verfügung.

Mit diesen Substanzen soll die autogene Knochenentnahme und -transplantation gänzlich vermieden werden (Abb.1). Die Ansprüche sind jedoch weiter gehend. So soll die Knochenheilung in Bezug auf die Menge des neugebildeten Knochens und in Bezug auf seine Qualität positiv beeinflusst werden. Idealerweise soll ebenso die Zeit, in der die Knochenheilung stattfindet, beschleunigt werden, so dass die Knochenersatzstoffe das autogene Knochentransplantat nicht nur eins-zu-eins ersetzen, sondern in biologischer Hinsicht übertreffen [9].

Um diese hoch gesteckten Ziele zu erreichen, müssen Knochenersatzstoffe zahlreiche Anforderungen aus einem umfangreichen Katalog erfüllen.

Für den klinischen Einsatz in Unfallchirurgie und Orthopädie gelten dabei folgende Grundvoraussetzungen:

- intraoperative Bearbeitungsmöglichkeit (Bohren, Sägen),

- sichere Defektauffüllung,

- sichere Applikation (kein Abschwimmen aus dem Defekt),

- mechanische Stabilität, 
- Gewebeverträglichkeit (kein Entzündungsreiz, keine lokalen oder systemischen Effekte des Materials bzw. seiner Abbauprodukte),

- Bioaktivität im Sinne der Definitionen

- Osteokonduktion (= Leitschieneneffekt)

- Osteoinduktion (= de novo Knochenentstehung), [1].

Neben diesen Grundvoraussetzungen erscheint es sehr wünschenswert, dass Knochenersatzstoffe als Trägermaterialien für Antibiotika, Wachstumsfaktoren und Zytostatika eingesetzt werden können, um sie gerade in den Situationen, die die Entstehung von Knochendefekten gefördert haben (Osteitis/Osteomyelitis, tumoröse ossäre Destruktion, delayed/ non-union), verwenden zu können.

Die Zahl der Substanzen, die für den klinischen Einsatz als Alternativen zum autogenen Knochen angeboten werden, ist sehr groß. Für eine Klassifikation kann man diese Substanzen in vier Gruppen aufteilen [8].

1. Substanzen anorganischen Ursprungs

2. Substanzen organischen Ursprungs

3. Metalle und

4. Komposite.

Diese Klassifikation ist insofern hilfreich, als dass sie die Effekte, mit der die Substanzen aus den einzelnen Gruppen bei der Behandlung von Knochendefekten aktiv werden, einbezieht.

\section{Kalziumphosphatverbindungen}

Zur Gruppe der anorganischen Knochenersatzstoffe zählen alle Kalziumphosphatverbindungen (nichtkeramisch, keramisch), Kalziumsulphate, Gläser/Glaskeramiken, Zemente auf Kalziumbasis und andere Mischungen (z.B. Trikalziumphosphat mit Kalziumsulfat).

Die letztgenannten Materialien werden synthetisch hergestellt oder aus bovinem Knochen bzw. Korallen gewonnen [7]. Entsprechend des Herstellungsprozesses unterscheiden sich die Kalziumphosphat-Keramiken in ihren stöchiometrischen Ca/P-Verhältnissen, Phasenreinheit, Porenvolumina, mechanischer Belastbarkeit, Oberflächenstruktur (Abb.2) und vielen anderen Punkten mehr [3; 4; 14].

Vor allem die ersten drei Punkte beeinflussen die biologische Reaktion am Implantationsort, d.h., ob die Substanzen eingebaut und umbaut werden und so-
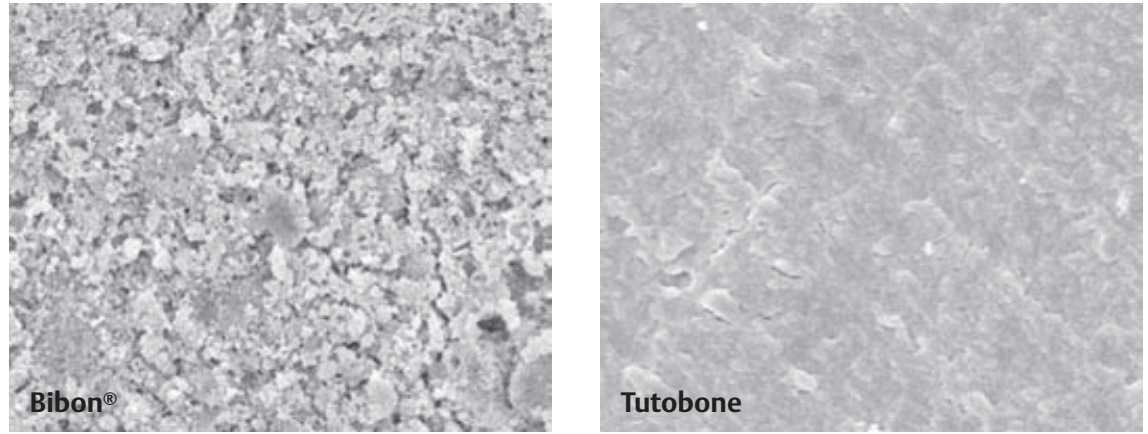

Abb.2 Rasterelektronenmikroskopie. $1000 \times$ Vergrößerung. Röhrenspannung 15 kv. Raue Oberfläche des Kalziumphosphatzementes $=$ Biobon $^{\circledR}$. Glatte Oberfläche eines Knochenderivates $=$ Tutobone $^{\circledR}$. Ob die unterschiedliche Oberflächenstruktur das Resorptionsverhalten von humanen Osteoklasten beeinflusst ist ungeklärt.

mit auf Dauer am Implantationsort verbleiben [9], oder ob sie sukzessive über humane Osteoklasten (Abb.3) abgebaut werden $[11 ; 12 ; 14]$ und den Wiederaufbau des Knochengewebes über Osteoblasten ermöglichen [5].

Trotz der ausgezeichneten Gewebeverträglichkeit der auf dem Markt angebotenen Substanzen bleiben einige Probleme, die den eins-zu-eins Ersatz von autogenem Knochen mit Kalziumphosphatverbindungen verhindern, bestehen.

Kalziumphosphatverbindungen - die am häufigsten eingesetzten sind Hydroxylapatit bzw. beta-Trikalziumphosphat sind immer azellulär. Sie sind niemals osteoinduktiv. In Abhängigkeit von dem Implantationsort und der Vitalität des Lagers dort (Knochen, Muskulatur, Narbengewebe) werden sie unterschiedlich rasch knöchern integriert bzw. aufgeschlüsselt [2;6], wobei das vaskuläre Einwachsen als der limitierende Faktor erscheint.
Sie sollten niemals diaphysär als „Stand alone-Implantat" verwendet werden, immer knöchern abgedeckelt sein, und bei der Verwendung als so genannter „Komposit-Graft", das heißt, bei der gemeinsamen Implantation mit zum Beispiel autogenem Knochen, sollte ein Mischungsverhältnis von $2 / 1=\mathrm{KES} /$ autogener Knochen nicht überschritten werden.

Gute Indikationen für den Einsatz von Kalziumphosphat-Keramiken als Formkörper, als Granulat, als Kalziumphosphatzement jeweils als „Stand-alone-Implantat" oder als Komposit sehen wir am:

1. Humeruskopf (Impressionsfraktur, z.B. Hill-Sachs-Defekt des Erwachsenen)

2. distaler Radius (begrenzte Indikationen), (u.a. 10), (Abb.4)

3. distaler Femur (Defektzonen, MIS)

4. Tibiakopf (ideale Indikation)

5. Pilon tibiale (Verhältnis KES/autogener Knochen = 1/1)

6. Kalkaneus (eingeschränkte Indikation).

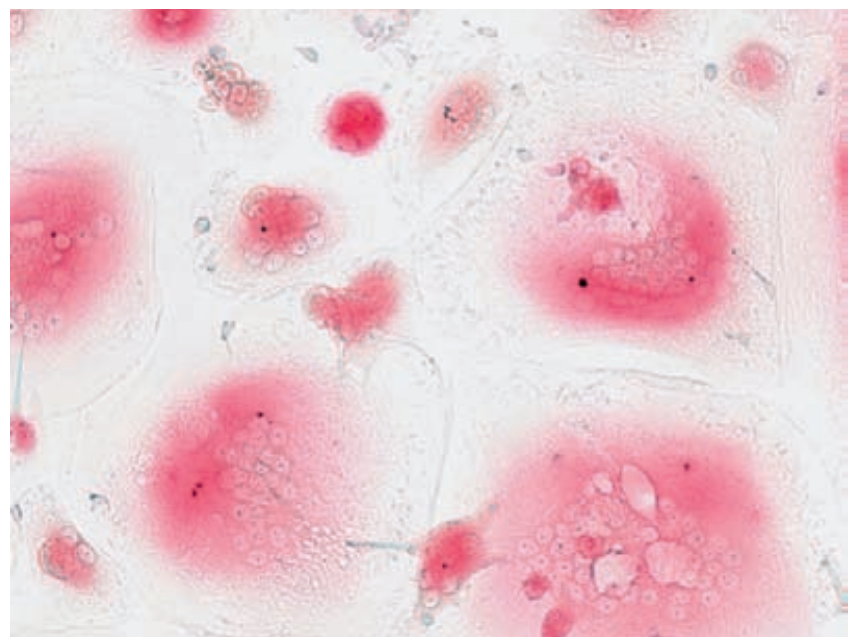

Abb. 3 Auflichtmikroskopie. TRAPFärbung. $20 \times$ Vergrößerung. Humane Osteoklasten in der Zellkultur. Multiple Zellkerne. 

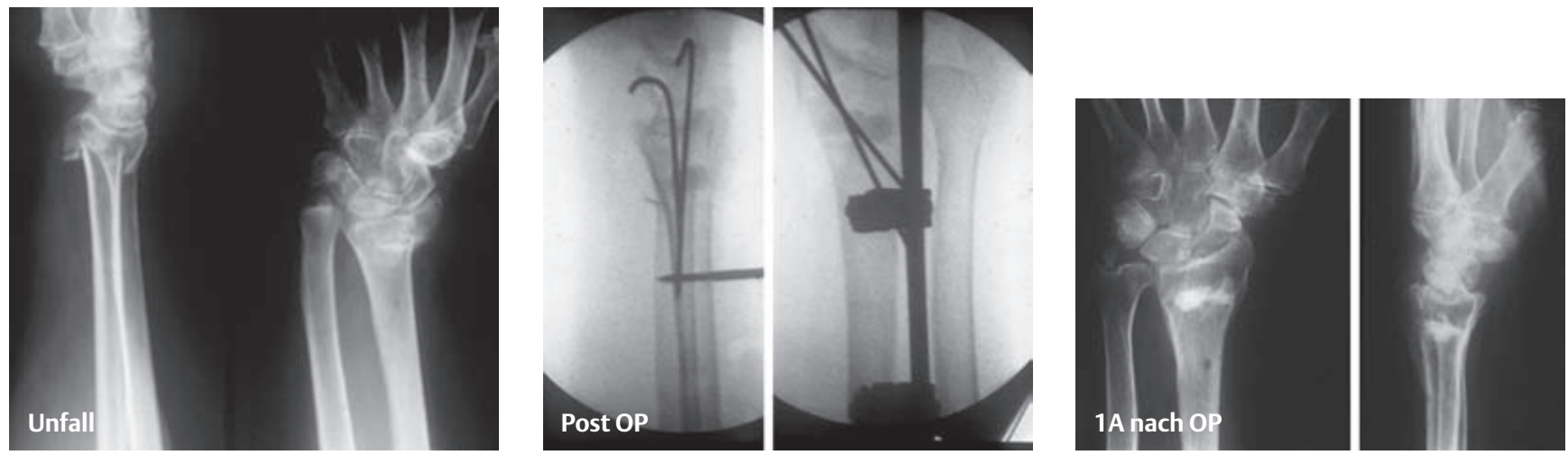

Abb.4 70-jährige Patientin. Distale Radiusextensionsfraktur. Fixateur-externe-Osteosynthese + 2 K-Drähte. Auffüllen des dorsalen Defektes mit Biobon $^{\circledR}$ Kalziumphosphatzement. Entstehung eines osteoimplantären Verbundes 1 A post OP. Freie Funktion. Keine Beschwerden.

Wir bevorzugen prinzipiell TCP-Keramiken als Formkörper oder als grobkörnige makroporöse Granulate, weil von einer höheren Resorptionsrate als bei der Verwendung von Hydroxylapatit auszugehen ist. Kalziumphosphatzemente setzen wir inzwischen nur bei kleineren Defekten am Tibiakopf bei B2-Frakturen, d.h. Impressionsfrakturen, die sich über ein kortikales Fenster aufstößeln lassen, für die Defektauffüllung ein.

In der Operationstechnik achten wir auf folgende Punkte (siehe u.a. 13):

1. nicht kontaminiertes/infiziertes Gebiet

2. großporige Keramik, möglichst als Formkörper

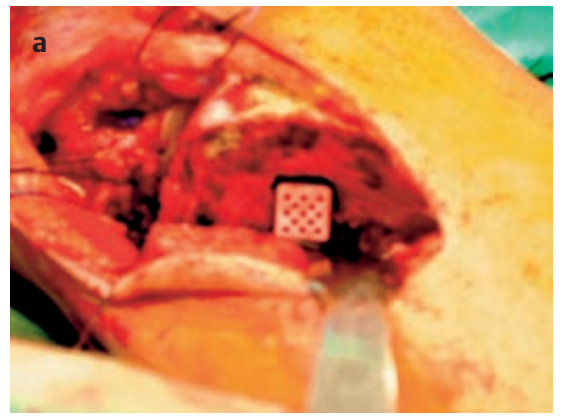

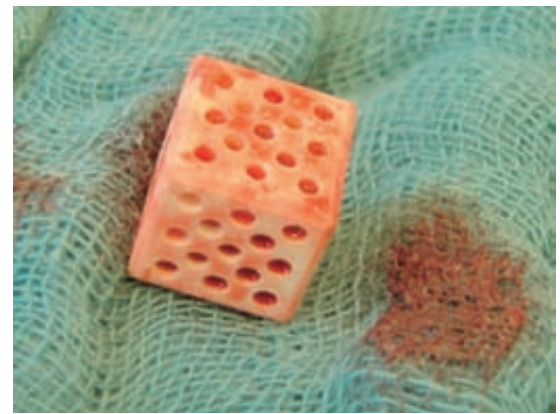

3. Knochenmarkbeimpfung bei „Standalone-Implantaten"

4. Mischungsverhältnis Knochenersatzstoff/autogener Knochen siehe oben.

5. Platzierung im Gelenkniveau: Immer subchondrale Lage mit - idealerweise - Spongiosa-Zwischenschicht zwischen Knorpel/subchondraler Lamelle und KES (z.B. Tibiakopfspalt-/Impressionsfraktur)

6. Abdeckeln durch Spongiosa bzw. Kortikalis, um einen direkten Kontakt zwischen dem in den Knochen eingebrachten KES und dem umgebenden Weichgewebe zu vermeiden (Abb.5a-d)

7. stabile Osteosynthese, wobei die mechanischen Eigenschaften auch der

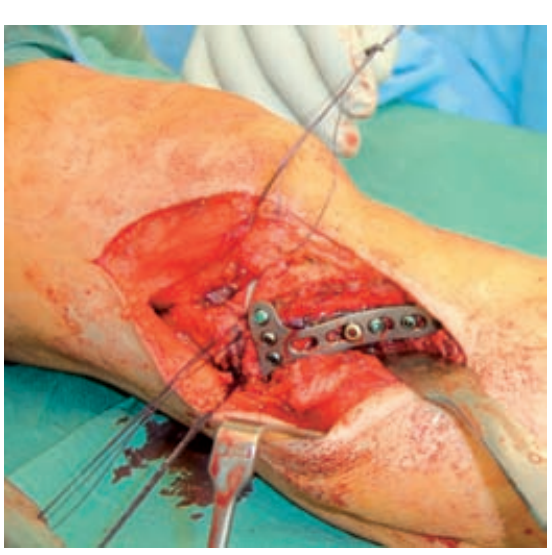

Formkörper so eingeschätzt werden müssen, als wären kortikospongiöse Chips transplantiert worden.

8. perioperative Antibiotika-Prophylaxe (Zweitgeneration Cephalosporin)

9. Krankengymnastik und Belastungsaufbau bzw. Röntgenkontrollen entsprechend der Standard-Schemata für die betroffene Region.

Beachtet man diese Regeln, dann liegt der große Vorteil der KalziumphosphatKeramiken vor allen Dingen darin, dass:

- ihre Anwendung die OP-Zeit verkürzt;

- idealerweise der Verzicht auf die Entnahme autogenen Knochens möglich ist;

- bei einer unzureichenden Menge des

Abb.5 (a) Fotografie, intraoperativ. Tibiakopffraktur. 52-jährige Patientin. Nach Anheben des Defektes Auffüllen mit Cerasorb ${ }^{\circledR}$ TCP Formkörper (b) Fotografie, intraoperativ. Abdeckeln des Fensters mit autogener Knochenlamelle. 3,5er-LCP Osteosynthese (c) Röntgenbilder vor Entlassung. Anatomische Rekonstruktion.
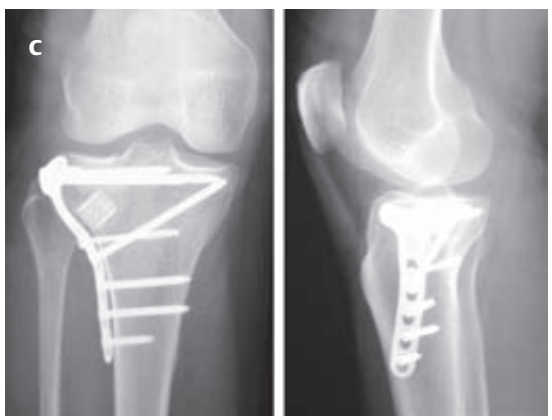


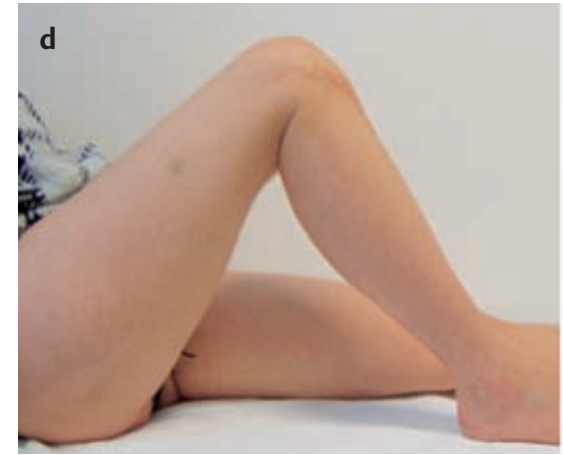

explantierten Knochens dessen Volumen bis auf das Dreifache durch einen Knochenersatzstoffe „gestreckt“ werden kann;

- sie komplikationsarm in der Anwendung bei Beachtung der oben aufgeführten Regeln sind.

Potenzielle Nachteile sind darin zu sehen, dass die Keramiken:

- biologisch weniger aktiv sind als autogener Knochen;

- die Beurteilung der Knochenbildung bei radiologischen Verlaufskontrollen erschwert ist, da die Keramiken sofort als röntgendichte Strukturen erscheinen;

- insbesondere Hydroxylapatit-Keramiken als Implantate dauerhaft reizlos

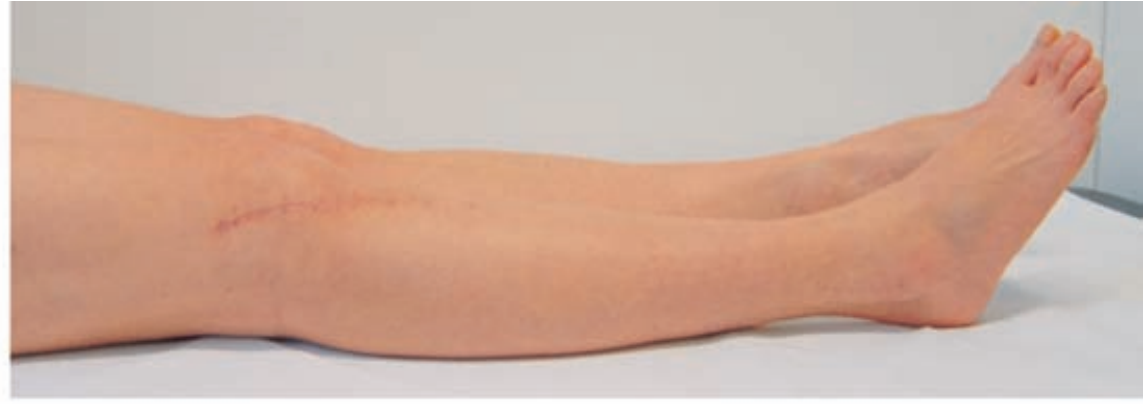

Abb.5 (d) Fotografie. Funktion im Verlauf.

als osteoimplantärer Verbund im Knochen zurück bleiben;

- Einzelbeobachtungen zeigen, dass selbst Jahre nach der Implantation und der Metallentfernung, Bagatelltraumen zu „Re“-Frakturen in den bzw. durch die eingebrachten Formkörper führen können, insbesondere dann, wenn keine vollständige knöcherne Abdeckelung der Keramiken gegen das Weichgebwebe gewährleistet war (Abb.6a und $\mathbf{b})$.

\section{Schlussfolgerung}

Kalziumphosphat-Keramiken (HA, TCP und Mischkeramiken aus HA und TCP bzw. beta- und alpha TCP) sind zuverlässig unter Beachtung bestimmter Kau-

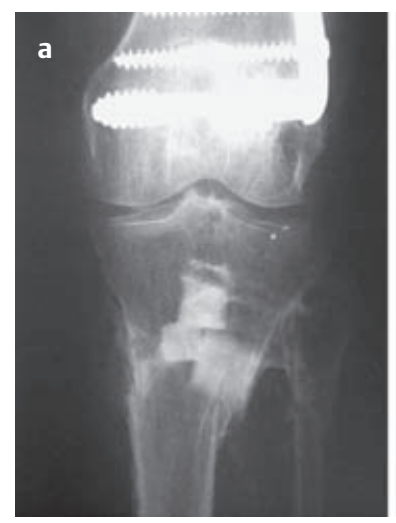

„Refraktur“ nach Bagatelltrauma
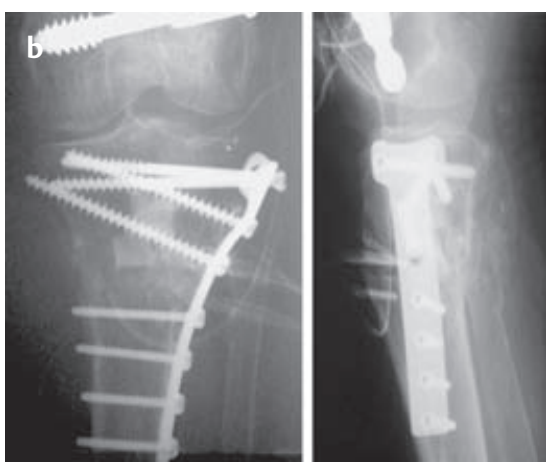

telen in der Unfallchirurgie, Orthopädie erfolgreich einsetzbar. Eine gewisse $\mathrm{Zu}-$ rückhaltung bei der Indikationsstellung ist nötig, um die Materialien in Bezug auf ihre biologische Potenz und ihre mechanischen Eigenschaften nicht zu überfordern, eine Überforderung, die zu unerwünschten Resultaten in Bezug auf die Fraktur- bzw. Defektheilung führen kann. Bei so genannten „bulk implants“, d.h. großen Mengen keramischer Substanzen muss eine ausreichende Biologisierung entweder über autogenen Knochen oder durch die simultane Applikation von Wachstumsfaktoren, z.B. BMPs erreicht werden. Gleichzeitig sind unter diesen Bedingungen eine sichere, vollständige Weichteilrekonstruktion mit gut durchblutetem Muskel/Muskel-Haut-Mantel und eine stabile Osteosynthese absolute Grundvoraussetzung für den Therapieerfolg.

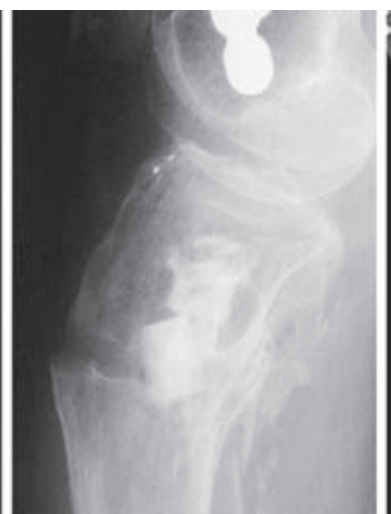

Abb.6 (a) Z.n. Tibiakopffraktur 1994. Primärversorgung mit Endobon ${ }^{\circledR}$ und Plattenosteosynthese. Z. n. ME. Jetzt: Nach Bagatelltrauma 2001: Fraktur „durch“ die Endobon ${ }^{\circledR}$ _ Keramik. (Pfeil: Randständiger Endobon ${ }^{\circledR}$ Block. (b) Therapie: Autogenes Spongiosatransplantat und 4,5er-T-Plattenosteo synthese nach Entfernung des randständigen Endobon ${ }^{\circledR}$-Blockes. 


\section{Literatur}

1 Albrektsson T, Johansson C Osteoinduction, osteoconduction and osseointegration. In: "The use of bone subtitutes in spine surgery. A state of the art review." Eds: Robert Gunzburg et al. Springer Heidelberg 2002; 12-17

2 Bauer TW, Mutschler GF. Bone graft materials: An overview of basic sciences. Clin Orthop Rel Res 2000; 371:10-27

3 Bohner M. Physical and chemical aspects of calcium phosphates used in spinal surgery. In: "The use of bone substitutes in spine surgery. A state of the art review." Eds: Robert Gunzburg et al. Springer Heidelberg 2002; 30-37

4 Epple M. Biomaterialien und Biomineralisation. Teubner Studienbücher Chemie 2003

5 Martin TJ, Sims NA. Osteoclast-derived activity in the coupling of bone formation to resorption. Trends Mol Med. 2005; 11: 76-81

6 Müller-Mai C. Bioaktive Granulate in der Unfallchirurgie. VNM Science Publishing München. 2003

7 Reif D, Leuner B, Hotz G. Temporäre Knochenersatzmaterialien auf der Basis von Calciumphosphat. In: „Biodegradierbare Implantate und Materialien." Eds: Lutz Claes et al. Hefte Unfallchirurgie 1997; 265: 270277

8 Rueger JM. Knochenersatzmittel. Hefte Unfallheilkunde 1992; 213

${ }^{9}$ Rueger JM. Knochenersatzmittel: Heutiger Stand und Ausblick. Orthopäde 1998; 27: 72-79

10 Sailer R, Gabl M, Lutz M, Seykora P, Pechlaner $\mathrm{S}$. Integration einer porösen Hydroxalapatitkeramik am distalen Radius älterer Patienten. Unfallchirurg 1999; 102: 532-534

11 Schilling AF, Linhart W, Filke S, et al. Resorbability of bone substitute materials by human osteoclasts. Biomaterials 2004; 25: 3963-3972

12 Schilling AF, Filke S, Brink S, Korbmacher H, Amling M, Rueger JM. Osteoclasts and Biomaterials. Eur J Trauma 2006; 32: 107-13

13 Shors EC. Bone graft substitutes. Clinical studies using coralline hydroxyapatite. In: "Biomaterials in Surgery." Eds. GHIM Walenkamp. Thieme. Stuttgart. 1998. 83-89

14 Yamada S, Heymann D, Bouler JM, Dalcusi G. Osteoclastic resorption of calciumphosphate ceramics with different hydoxyapatite/betatricalciumphosphate ratios. Biomaterials 1997; 18: 1037-1041
Dr. med. Johannes M. Rueger, Direktor

Dr. med. Andreas Rücker,

Oberarzt

Dr. med. Arnd Schilling,

Wiss. Assistent

Dr. med. Daniel Briem

Oberarzt

Klinik für Unfall-, Hand- und

Wiederherstellungschirurgie

Operatives Zentrum

Universitätsklinikum Hamburg

Eppendorf

Martinistraße 52

20246 Hamburg 